\title{
Enonciation et argumentation dans les rapports parlementaires autour du gaz de schiste : impartialité ou influence?
}

\author{
Michele Monte ${ }^{1}$ \\ Laboratoire Babel - EA 2649 - Université de Toulon
}

\begin{abstract}
Résumé. L'article étudie le fonctionnement énonciatif et argumentatif de trois rapports parlementaires. Assumant pleinement leur rôle de prescripteurs de l'action législative, les rapporteurs affichent un éthos de conviction mais s'attachent aussi à montrer leur impartialité. Dans leur argumentation sur les risques environnementaux du gaz de schiste, ils débattent de la fiabilité des chiffres et construisent une schématisation distincte d'un rapport à l'autre tant du point de vue du lexique que des autorités invoquées. L'article éclaire ainsi les processus langagiers par lesquels s'articulent dans le genre du rapport l'information et la préconisation.
\end{abstract}

Cette communication se propose d'éclairer l'étude du fonctionnement énonciatif et argumentatif des rapports parlementaires en prenant comme corpus les trois rapports produits en 2011 au moment du débat national sur l'exploitation du gaz de schiste. Lors d'un travail précédent, à dominante quantitative, appuyé sur la textométrie, où nous avons comparé des rapports parlementaires, scientifiques et techniques (Ledouble et Monte 2019), les spécificités énonciatives des rapports parlementaires nous ont paru assez fortes pour mériter d'être étudiées de façon plus approfondie. Notre communication comportera trois parties : dans la première, nous exposerons les circonstances de rédaction des rapports et les caractéristiques de notre corpus. Dans la deuxième, nous étudierons les dispositifs énonciatifs et chercherons à caractériser un éthos parlementaire. Enfin, dans la troisième partie, nous nous attacherons à quelques aspects du fonctionnement argumentatif de ces textes en contrastant les trois rapports entre eux.

\section{Contexte et choix du corpus}

\subsection{Contexte et situation de communication}

Une vive controverse sur le gaz de schiste a agité la France en 2010 et 2011 lorsque l'opinion publique a eu connaissance de permis d'exploitation accordés à des sociétés pétrolières dans des régions du Sud-Est du Massif central, régions très touristiques et pauvres en eau alors que l'exploitation par fracturation hydraulique exige d'immenses ressources hydriques et présente des risques de pollution des sols importants. La contestation populaire a amené les parlementaires des deux bords à formuler des propositions de loi visant à interdire l'exploitation et le gouvernement Fillon à soutenir la proposition Jacob par l'adoption de la procédure d'urgence. La loi est votée le 11 mai 2011 à l'Assemblée Nationale et, dans une version légèrement amendée, au Sénat le 9 juin 2011, puis, après la réunion de la commission mixte parlementaire, adoptée en deuxième lecture 
respectivement les 21 et 30 juin et promulguée le 13 juillet ${ }^{1}$. Elle « interdi[t] l'exploration et l'exploitation des mines d'hydrocarbures liquides ou gazeux par fracturation hydraulique et abrog[e] les permis exclusifs de recherches comportant des projets ayant recours à cette technique " mais elle autorise des explorations à but scientifique et n'écarte pas la possibilité d'exploiter le gaz de schiste au cas où des procédés moins agressifs pour l'environnement seraient mis au point ${ }^{2}$.

Comme c'est souvent le cas, le vote de la loi a été précédé par la production de rapports ${ }^{3}$ qui peuvent être envisagés comme des instruments d'action publique (Chailleux 2019) :

- Un rapport commandité le 4 février 2011 par la ministre de l'écologie et du développement durable et le ministre de l'industrie et de l'énergie charge le viceprésident du Conseil général de l'industrie, de l'énergie et des technologies (CGIET) et le vice-président du Conseil général de l'environnement et du développement durable (CGEDD) de diligenter une mission portant sur les hydrocarbures de rochemère. Ce rapport (désormais CGIET-CGEDD) rédigé par des ingénieurs du corps des mines, des ponts, des eaux et des forêts, est publié le 21 avril 2011, juste avant l'examen de la loi, et sera suivi en février 2012 d'un rapport complémentaire.

- La commission du développement durable et de l'aménagement du territoire de l'Assemblée Nationale a par ailleurs mandaté le $1^{\text {er }}$ mars une mission d'information bipartisane qui était chargée de « compléter la vision technique des experts mandatés par le Gouvernement » (p. 8) et de rendre un rapport sous trois mois. MM. Gonnot et Martin ont rendu leur rapport (désormais GM) le 8 juin 2011, mais entretemps, pour couper court à l'émotion de l'opinion publique et peut-être pour faire voter une loi minimaliste qui préservait les intérêts à long terme des industriels, le gouvernement avait enclenché la procédure d'urgence et la loi était en cours d'adoption.

- L'arrivée à l'ordre du jour de l'Assemblée Nationale du vote de la loi a du coup amené sa commission du développement durable et de l'aménagement du territoire à mandater deux de ses membres, MM. Havard et Chanteguet, qui produisent un rapport (désormais HC) chargé d'évaluer la proposition de loi et éventuellement de la modifier avant qu'elle soit soumise au vote : il est rendu le 4 mai 2011.

- Parallèlement, le Sénat charge un de ses membres, M. Houel, de produire un rapport (désormais $\mathrm{H}$ ) chargé d'évaluer les deux propositions de loi et la loi votée en première lecture à l'Assemblée Nationale : il est rendu le 25 mai 2011.

Ultérieurement trois autres rapports d'information sur le gaz de schiste seront produits :

- en novembre 2013, MM. Lenoir et Bataille, membres de l'Office Parlementaire d'Évaluation des Choix Scientifiques et Technologiques (OPECST), remettent un rapport (désigné infra par OPECST) portant sur « les techniques alternatives à la fracturation hydraulique $»$;

- en avril 2014, M. Barbier remet un rapport (désormais B) sur « l'impact économique de l'exploitation des gaz de schiste»;

- en mai 2015, MM. Bataille et Schneider présentent un rapport d'information sur « les nouvelles données de la géopolitique de l'énergie : pétrole et gaz naturel» (désormais BS).

Parallèlement nous disposons de deux rapports scientifiques :

- l'un a été élaboré à la demande du conseil régional d'Ile-de-France et déposé en mars 2012. Il s'intitule « Risques potentiels de l'exploration et de l'exploitation des hydrocarbures non conventionnels en Ile-de-France » et sera désigné par CSIDF ;

- l'autre a été rendu public par le Conseil des académies canadiennes en 2014 et s'intitule «Incidences environnementales liées à l'extraction du gaz de schiste au Canada » (il sera désigné par CAC).

Dans notre précédent travail, nous avons mené une étude outillée essentiellement quantitative visant à dégager les thématiques et les principales caractéristiques énonciatives de ce corpus. Nous avons notamment mis en relief des différences liées à la date de production des rapports, à leurs sources, à leurs objectifs. Mais il nous a semblé intéressant d'approfondir l'analyse des rapports parlementaires de 2011 qui se situent dans le contexte de crise rappelé plus haut et dont l'originalité nous est apparue à plusieurs reprises. Le présent article s'inscrit dans des interrogations plus générales sur le traitement de la 
conflictualité dans l'espace public (Lemieux 2007, Rennes 2016).

\subsection{Caractéristiques du corpus et problématique}

Nous disposons donc pour l'année 2011 de trois rapports parlementaires de nature différente. Deux d'entre eux ( $\mathrm{HC}$ et $\mathrm{H})$ préparent le vote de la loi et sont chargés de justifier les préconisations de la commission relativement aux amendements déposés par les députés. Ils comprennent chacun trois parties : une partie sur les enjeux de l'exploitation du gaz de schiste, une partie sur les objectifs de la loi et une partie sur le travail de la commission (discussion générale et examen des articles). Le troisième rapport (GM) est le résultat d'une mission d'information et ne présente qu'un état des lieux des informations disponibles et des préconisations concernant à la fois l'exploitation du gaz de schiste et la réforme du code minier. Pour les comparer valablement, nous avons exclu des rapports les débats en commission. Les parties étudiées sont donc homogènes du point de vue textuel : les rapporteurs font un état des connaissances et émettent des préconisations, et, sur le plan de la typologie textuelle (Adam 2011), le texte est donc à la fois explicatif et argumentatif. Sur le plan quantitatif, en revanche, les différences sont grandes : la partie retenue d'HC comporte 8193 occurrences $^{4}$ (23 pages), celle d'H 6881 occurrences (19 pages), alors que celle de GM en contient 31767 (100 pages). Ce dernier rapport est comparable quantitativement au rapport des ingénieurs du CGIET-CGEDD qui comporte 28317 occurrences (73 pages).

Notre travail antérieur (Ledouble et Monte 2019) a mis en relief, pour ces trois rapports ainsi que pour le rapport de l'OPECST de 2013, des spécificités énonciatives qui les distinguent des rapports scientifiques, d'une part, et des deux autres rapports parlementaires, d'autre part. Pour résumer grossièrement ces premiers résultats, on peut dire que la nature argumentative de ces rapports est plus affirmée et que les parlementaires ne craignent pas de s'y engager. Nous voudrions donc à présent caractériser plus finement l'éthos de ces rapports puis nous intéresser à la façon dont s'y articulent information et préconisation.

\section{Un éthos parlementaire singulier}

À la suite d'Amossy (2010), nous entendons par éthos l'image de soi construite par le discours : il s'agit pour nous d'un concept interprétatif qui repose sur la prise en compte d'une grande variété de traits linguistiques. Comme l'indique Maingueneau (2002), même un texte écrit peut posséder un éthos car les actes de langage, le lexique, la syntaxe, les choix énonciatifs et rhétoriques le dotent d'un ton qui permet au lecteur de caractériser l'image de soi du locuteur, au moins en la rattachant à des types généraux (ton professoral, amical, décontracté, etc.) Nous envisageons donc l'éthos comme un élément de l'argumentation qui assoit - ou éventuellement dessert, s'il est mal maitrisé - la crédibilité des locuteurs. Nous nous proposons de l'appréhender à partir de l'observation de traits énonciatifs et lexicaux caractéristiques de tout ou partie de ces trois rapports que nous comparerons entre eux et avec l'ensemble de notre corpus.

\subsection{Un éthos de conviction}

De nombreux discours institutionnels sont caractérisés par un évitement de la polémique : Oger et Ollivier-Yaniv (2006) parlent de « lissage discursif », Oger (2013) d'« institution 
du neutre ». Le rapport, quant à lui, que l'on peut caractériser comme un «macro- genre » destiné à « dire ce qui est à l'intention d'un destinataire intéressé » (Née, Oger, Sitri 2017 : 11), allie une double visée informative et prescriptive, ce qui peut conduire à un engagement plus explicite du scripteur selon le dispositif institutionnel dans lequel est pris le rapport (voir, pour les rapports éducatifs, Née, Sitri et Veniard 2014). Au sein du macro- genre " rapport ", il nous semblait donc important de dégager les caractéristiques énonciatives du rapport d'information en contexte politique et d'évaluer l'homogénéité ou l'hétérogénéité du corpus. Le calcul des spécificités effectué par le logiciel TXM nous a permis d'observer que le lemme « rapporteur » est nettement surreprésenté ${ }^{5}$ dans certains rapports ${ }^{6}$ :

Fig. 1. Spécificités du lemme « rapporteur » dans le corpus global

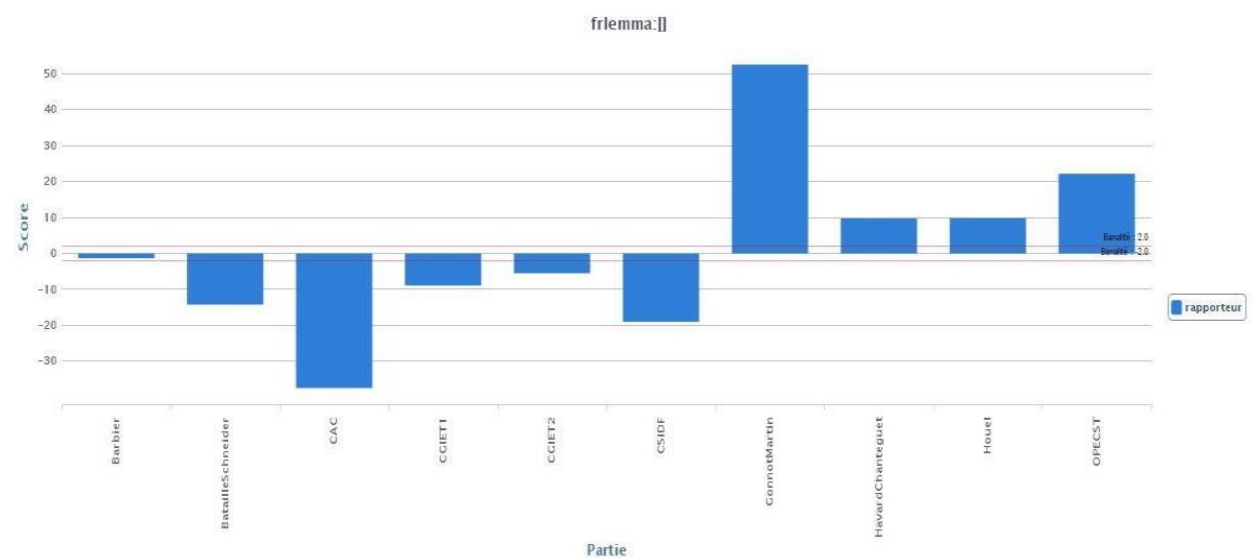

L'observation des emplois en contexte montre que le syntagme votre/vos rapporteur(s) est totalement absent des rapports des ingénieurs et scientifiques. Dans les rapports parlementaires, sa fréquence permet de contraster les rapports de 2011 et 2013 (OPECST) et ceux de de 2014 et 2015 : GM l'emploie 114 fois là où $\mathrm{BS}$, qui comporte deux fois plus d'occurrences, ne l'utilise que 17 fois. $\mathrm{HC}$ et $\mathrm{H}$ présentent respectivement 26 et 24 occurrences. Les autres rapports préfèrent les syntagmes la mission, la commission, les tournures passives ou le on inclusif.

Si l'on s'intéresse au contexte du syntagme, on observe que les rapporteurs se présentent soit dans leur mission d'enquête et d'audition (ex. 1), soit dans leur rôle qui est d'éclairer les décisions de leurs collègues (ex. 2 et 3 où nous avons souligné les termes évaluatifs) :

1. Néanmoins, au cours de leurs auditions, vos rapporteurs ont constaté que, pour chaque personne auditionnée, l'expression « non conventionnel » recouvrait la même réalité. (HC, p.11)

2. Votre rapporteur considère, aussi bien par son expérience de terrain qu'à la suite des auditions qu'il a menées, que l'émotion considérable suscitée par les projets d'exploration des gaz et huiles de schiste montre clairement l'inadaptation des procédures employées. (H, p.20)

3. Cette modalité apparaît largement insuffisante à vos rapporteurs. (GM, p.81)

La première catégorie d'exemples est plus abondante chez GM, qui avaient une mission d'information et ont pu effectuer plusieurs déplacements. La seconde est également répartie dans les trois rapports et révèle un fort engagement des parlementaires en 2011. Nous avons relevé parmi les verbes dont le syntagme est le sujet beaucoup de verbes d'opinion, de souhait ou de conseil ${ }^{7}$ : considérer (11), constater (11), estimer (7), recommander (6), juger (5), souligner (5), être surpris (4), observer (3), plaider (3), souhaiter (3), douter (2), regretter, s'inquiéter, (dé)conseiller. Comme le montrent l'exemple 2 et, plus bas, l'exemple 9, les verbes d'opinion introduisent des jugements nettement formulés qui préparent la décision. Ils introduisent parfois des actes proprement directifs, comme en (4). Quant aux verbes directifs explicites (ex. 5), ils illustrent le volet prescriptif du rapport : 
4. Vos rapporteurs sont d'avis qu'il faut renforcer l'association des citoyens à la prise de décision publique dès la délivrance des permis de recherches. (HC, p.28)

5. Vos rapporteurs recommandent que l'administration veille à ce que tout chantier pétrogazier ouvert sur le territoire national dispose à sa proximité immédiate d'équipements de secours et de lutte antipollution. (GM, p.56)

Les députés assument pleinement l'autorité qui leur est dévolue par leurs collègues. Toutefois, il faut préciser que, si le syntagme votre/vos rapporteur(s) est très spécifique des trois rapports que nous nous proposons d'étudier, les verbes qui l'accompagnent et que nous venons de commenter sont présents dans tous les rapports et ne sortent pas de la zone de banalité ${ }^{8}$. En effet, ce qui varie, c'est le sujet qui régit ces verbes : à côté de votre/vos rapporteur(s) de $\mathrm{HC}, \mathrm{H}$, GM et OPECST, on trouve aussi la mission de CGIET, on du CSIDF, le comité d'experts du CAC. Les évaluatifs et directifs sont une caractéristique commune de ce genre de rapports, et seul varie l'embrayage énonciatif. L'emploi répété de votre/vos rapporteur(s) est-il une routine ${ }^{9}$ de ces rapports parlementaires ou $\mathrm{HC}, \mathrm{H}$ et GM ont-ils voulu, dans un contexte de contestation populaire, affirmer leur engagement au service de la Nation? Il est difficile de trancher faute d'une comparaison avec un corpus plus vaste. Ce que nous observons en tout cas, c'est qu'une marge de choix existe puisque le rapport BS, lui, évite soigneusement toutes les formes déictiques, y compris le on, et évite même le mot mission (spécificité négative de 11,5). La formulation des évaluations est ainsi très différente entre les rapports de 2011 et celui de $2015^{10}$ :

6. Devant ces éléments, vos rapporteurs sont particulièrement dubitatifs, et même réticents, devant la perspective d'une exploration gazière réalisée sur ces territoires. [...] Ainsi, l'interdiction de la fracturation hydraulique laisse demeurer les hydrocarbures dans le sous-sol qui les abrite. Vos rapporteurs s'accordent pour considérer qu'il s'agit, pour l'heure, au vu des incertitudes de la technique et des défaillances de la législation, de la meilleure solution. (GM, p.60)

7. En Europe, la France partage avec la Bulgarie la particularité d'avoir interdit la fracturation hydraulique. Pour la Bulgarie, cette interdiction est interprétée comme le résultat d'une proximité traditionnelle avec la Russie. Pour notre pays, c'est totalement incompréhensible. Son image, associée à Descartes, aux Lumières et à la pensée rationnelle, en pâtit largement. (BS, p.239)

Alors que chez BS, les jugements de valeur s'expriment sous la forme d'un énoncé avec modus intégré où la source du point de vue est effacée, chez les rapporteurs de 2011, le modus et le dictum ${ }^{11}$ sont séparés et le point de vue peut sans hésitation être rapporté aux auteurs du rapport. On ne trouve chez BS que trois occurrences de les rapporteurs (notons l'emploi de l'article défini plutôt que du possessif) en position d'évaluateurs et sur des questions qui ne concernent jamais la France : « les rapporteurs tiennent à observer », "les rapporteurs n'ont pas perçu la volonté de désengagement des États-Unis de cette région du monde ». Les autres emplois, également rares, concernent les auditions (« toutes les personnes entendues par les rapporteurs »). On peut y voir une stratégie, consciente ou non, d'effacement énonciatif pour se donner un éthos d'objectivité alors même que le rapport conclut fortement en faveur d'une exploration des gaz de schiste dans le sous-sol français.

À l'inverse, les rapporteurs de 2011 préfèrent endosser pleinement leur mission d'éclaireurs du débat public, ce qui les conduit à adopter un éthos de conviction et à assumer personnellement les évaluations contenues dans le texte. Nous associons ce trait au contexte de crise en 2011, mais c'est peut-être BS qui s'écarte d'une norme énonciative commune à tous les rapports parlementaires.

À l'intérieur du corpus de 2011, GM expriment leurs prescriptions plus directement que $\mathrm{HC}$ et $\mathrm{H}$ : ce sont eux qui utilisent le plus les verbes recommander, plaider, (dé)conseiller, sans toutefois sortir de la zone de banalité. Le verbe convenir dans son sens prescriptif est par ailleurs sur-employé dans leur rapport (spécificité positive $+3,7$ ) :

8. Il convient de tirer les leçons de ces dysfonctionnements, d'autant qu'ils ne sont en rien imputables à une quelconque violation de la réglementation en vigueur par les entreprises ou par l'administration. (GM, p.74) 
Il y a là un certain paradoxe, puisque cette mission a rendu son rapport alors que la loi avait déjà été votée en première lecture à l'Assemblée Nationale, ce que les rapporteurs déplorent, en déclarant qu'il « ne [leur] a pas paru cohérent, ni même logique, que le Parlement puisse définitivement adopter une loi avant d'entendre le rapport de ses membres sur le sujet traité » (p.11). La forte dimension prescriptive peut dès lors s'expliquer par l'insatisfaction de GM face aux mesures prises et par leur désir de fournir au Parlement des raisons de rouvrir le débat afin de légiférer dans le calme et de façon plus définitive ${ }^{12}$.

\subsection{Un éthos d'impartialité}

Si les rapporteurs font preuve de conviction, ils veillent également à cultiver un éthos d'impartialité, qu'attestent une série de traits que nous allons à présent examiner.

Chez $\mathrm{HC}$, le premier de ces traits est l'emploi fréquent de néanmoins (spécificité positive $+4,2)^{13}$ :

9. Vos rapporteurs comprennent en ce sens la tentation que représente la découverte soudaine de réserves d'hydrocarbures dans le sous-sol français. Néanmoins, ils considèrent qu'en dépit des estimations annoncées, il convient de revenir sur les autorisations accordées dès lors que leurs titulaires envisagent de recourir à la fracturation hydraulique, et d'engager la modernisation de la législation en vigueur, au service d'une politique énergétique nationale claire et ambitieuse. (HC, p. 23)

Selon notre analyse, dans un énoncé « A néanmoins B », les deux points de vue $p$ et $q$ opposés par le concessif sont mis sur le même plan, le mouvement concessif est conçu d'emblée dans sa totalité et « la situation est envisagée globalement sous ses deux aspects contradictoires » (Mellet et Monte, 2008 : 72). L'inférence que l'on pourrait tirer du contenu $p$ de l'énoncé A est invalidée par le contenu $q$ de $\mathrm{B}$, mais $p$ n'est pas contesté pour autant, ce qu'exprime très bien (9) où les rapporteurs témoignent de leur compréhension vis-à-vis de $p$. On a donc affaire à un connecteur concessif qui s'efforce de maintenir un certain équilibre entre les deux points de vue et qui convient parfaitement à la quête d'impartialité des parlementaires.

Chez GM, la quête d'impartialité se marque par un sur-emploi très net du verbe sembler $(+13)$ :

10. Il semble ainsi que des pratiques particulièrement déplorables aient eu cours dans les premiers temps de l'industrie. La formidable croissance de l'activité a généré des comportements particulièrement néfastes à l'environnement, que Gasland ne manque pas de souligner [...] Les investigations conduites par vos rapporteurs ont montré que nombre de ces dérives semblent révolues aujourd'hui. (GM, p.40)

Le verbe sembler permet à la fois d'atténuer des critiques virulentes tout en les formulant et de réserver le jugement sur des points sensibles.

Les trois rapports insistent d'autre part sur les nombreuses consultations qu'ils ont effectuées pour entendre différents points de vue. Houel écrit dans son introduction :

11. Il [votre rapporteur] a toutefois reçu de nombreux experts, industriels et acteurs environnementaux afin d'étayer solidement l'analyse de la proposition de loi qu'il propose dans le présent rapport. (H, p.6)

La liste des auditions figure à la fin de chaque rapport et les controverses entre industriels et défenseurs de l'environnement ou scientifiques sont clairement énoncées, avec une référence commune au film Gasland et à des études scientifiques contestant les affirmations des industriels :

12. En particulier, la contamination de l'eau potable par du gaz de schiste, qui constitue l'un des points centraux du film documentaire Gasland, a été présentée comme "impossible » par les industriels, qui mettent en avant l'épaisseur des couches géologiques qui séparent la rochemère des nappes souterraines d'eau potable. Une étude scientifique récente a pourtant $m i s$ en évidence des cas de contamination de l'eau potable qui semblent liés à l'exploitation du gaz de schiste. (H, p.13)

13. Il ne s'agit pas, pour vos rapporteurs, de se livrer à la critique d'une œuvre cinématographique 
[Gasland]. S'il fallait la mentionner eu égard à son influence décisive sur le débat, il convenait aussi d'en vérifier le contenu auprès des experts rencontrés à l'occasion des auditions. Les accusations de manipulation lancées par les industriels ont été accueillies avec scepticisme ; certains cependant ont avancé des explications convaincantes qui éclairent d'une lumière nouvelle l'exemple américain et mettent en perspective les risques encourus en cas d'exploitation sur le territoire français. (GM, p.39)

On voit sur ces deux exemples que le discours autre ${ }^{14}$, très important, est, sur le plan modal, intégré énonciativement au dire du locuteur premier par une description métalangagière catégorisante suivie, la plupart du temps, d'une paraphrase plutôt que d'une monstration (Authier-Revuz 2020). Les syntagmes qui le décrivent sont très variés, centrés sur le contenu des arguments (contamination), leur valeur illocutoire (accusation, explications), ou leur effet perlocutoire aux yeux des rapporteurs (convaincantes). Industriels et scientifiques sont les deux sources les plus citées :

14. L'exploitation des hydrocarbures liquides ou gazeux par des forages suivis de fracturation hydraulique comporte de nombreux risques. Au cours de leurs auditions, vos rapporteurs ont parfois eu le sentiment que les industriels balayaient nombre d'entre eux d'un revers de main. (HC, p.17)

15. Des experts et industriels auditionnés par votre rapporteur ont fait part de pistes pour assurer une exploitation des gaz de schiste plus respectueuse de l'environnement. Ces pistes restent à valider scientifiquement et techniquement. (H, p.14)

16. Vos rapporteurs constatent que la science n'écarte pas qu'un lien puisse exister entre l'élévation du taux de méthane dans les eaux de Pennsylvanie et le formidable développement de l'industrie des gaz de schiste qu'a connu cet État. [...] En l'absence de connaissance claire, la prudence impose l'application du principe de précaution et la réalisation d'enquêtes plus poussées. (GM, p.57)

Les exemples ci-dessus montrent que ces discours autres sont convoqués à diverses fins. En (14), il est simplement fait allusion aux propos des industriels, pour les critiquer. En (15), H s'appuie sur la même forme très résumée de RDA (ont fait part de) pour demander la poursuite des explorations scientifiques. La conclusion est inverse chez GM en (16) : ils s'appuient sur une étude dont ils ont résumé préalablement les conclusions pour réclamer la mise en œuvre du principe de précaution. Mais on aura noté que le point commun des trois rapports est leur soin à assortir les discours représentés de réserves sur leur validité. Dans un contexte mouvementé, auquel ils se réfèrent expressément avec des lexèmes différents « contestations » pour $\mathrm{HC}$, « mouvement populaire » pour $\mathrm{H}$, « manifestations populaires » et " polémique » pour GM -, il est nécessaire qu'ils construisent soigneusement leur éthos d'impartialité pour ne pas être pris à parti par les industriels ou les environnementalistes. Dans ce processus, l'appui sur la science et la méfiance vis-à-vis des industriels fonctionnent comme garants d'objectivité. Il se pourrait cependant que ce recours aux scientifiques soit plus un affichage qu'une réalité. En effet Chailleux qui a comparé les références bibliographiques de rapports français et québécois constate :

Les rapports parlementaires français (Gonnot-Martin et Havard-Chanteguet) font principalement référence à des documents administratifs et légaux pour supporter leurs conclusions et les sources scientifiques académiques sont assez rares. (Chailleux $2016: 542$ ) 
De nombreux documents sont également fournis par l'industrie gazière, ce qui produit un cadrage du problème public peu propice à l'impartialité. Chailleux souligne à juste titre que, dans cette controverse, c'est le débat public plutôt que les connaissances mobilisées par les rapports qui a pu infléchir l'action gouvernementale, même si la restriction au seul problème de la fracturation hydraulique résulte d'une volonté de la commission HavardChanteguet. Il nous semble cependant important d'étudier plus en détail l'argumentation déployée dans ces rapports, ce qui nous amènera à nuancer quelque peu les affirmations du sociologue tout en éclairant les processus généraux par lesquels se manifeste la dimension prescriptive du genre.

\section{Quelques stratégies argumentatives}

Même si GM ont pu aller enquêter sur le terrain aux USA contrairement aux autres, les cinq parlementaires rédacteurs de ces rapports disposent à peu près des mêmes informations qu'ils exposent en des termes apparemment proches, affichant des éthos similaires de conviction et d'impartialité. Pourtant les conclusions de leurs rapports sont différentes et sont l'aboutissement de mouvements argumentatifs distincts. Faute de place, nous limiterons ici notre démonstration à trois aspects : les titres, les données chiffrées mobilisées par les rapports et la façon dont est convoqué le respect de l'environnement. Cela nous permettra de mettre en évidence la manière dont les rapporteurs présentent les données en fonction de leurs objectifs et peuvent ainsi influencer leurs destinataires.

\subsection{Les titres}

L'organisation des trois rapports comporte de grandes similitudes. Les parlementaires résument les données techniques et économiques, rappellent la conjoncture internationale, puis passent en revue les " risques environnementaux et sanitaires intrinsèques à l'utilisation de la fracturation hydraulique »(HC) avant de s'intéresser aux aspects obsolètes du code minier et d'appeler le parlement « à se resaisir de la définition de la politique énergétique » $(\mathrm{HC})$. Cette démarche commune aux trois rapports est résumée sobrement par les titres de GM :

17. I. Les bases du débat : définitions techniques, éléments économiques et situation internationale - II. L'enjeu du débat : hydrocarbures de schiste et préservation de l'environnement - III. Les moyens du débat : les imperfections du code minier

Les titres des grandes parties, dans les trois rapports, se caractérisent par la même neutralité. Mais dès les sous-titres, se dessinent des orientations bien différentes dont rendent bien compte les concepts de schématisation et d'éclairage, portés par Grize :

Une schématisation donne à voir, mieux à regarder, une situation dans laquelle se trouvent des objets et des acteurs sous un certain éclairage. [...] Les objets du discours doivent être éclairés, ce qui revient à mettre en évidence quelques-unes de leurs facettes et à en occulter d'autres (Grize 2004 : 35 ; 40)

La schématisation est pour Grize la façon dont l'énonciateur A construit par le discours pour son destinataire $\mathrm{B}$ une représentation du monde qui tient compte de la finalité de $\mathrm{A}$, de l'image qu'il se fait de lui-même et de B, de sa vision de la situation et de ce dont il parle. Argumenter consiste à produire un discours exprimant cette représentation " en vue d'intervenir sur l'attitude, l'opinion et le comportement de quelqu'un » (2004: 35). Ainsi, en ce qui concerne l'estimation des réserves françaises en gaz de schiste, peut-on lire les sous-titres suivants :

18. Les pays européens séduits par un rêve de réduction de la dépendance énergétique (HC, p.15)

19. Une ressource inespérée pour l'indépendance énergétique (H, p.7)

20. Des ressources à confirmer (GM, p.34) 
En (18) « séduits » et surtout « rêve » sous-entendent que les pays européens se bercent d'illusions, (20) exprime de la prudence, alors qu'en (19) « inespérée » donne de la réalité à la ressource et que le syntagme "indépendance énergétique " fixe un horizon plus prometteur qu'une simple " réduction de la dépendance énergétique ». On peut aussi observer que le rapport Houel, le plus favorable à l'exploitation, intitule sa partie sur les risques « Le risque d'atteintes durables à l'environnement en cas d'utilisation de techniques inappropriées », ce qui présente les risques comme découlant d'un mauvais usage des techniques, alors qu'HC les qualifie d' "intrinsèques » et que GM optent pour des titres interrogatifs, ce qui oblige le lecteur à lire le rapport pour connaitre leur conclusion.

\subsection{Le débat autour des chiffres}

Dans les développements, les différences s'accusent. Là où $\mathrm{HC}$ « tiennent à souligner que les ressources estimées sur le continent européen sont très faibles au regard des évaluations de l'Agence internationale de l'énergie pour les autres continents » (p.16) et incluent un tableau de l'AIE à l'appui de leurs dires, $\mathrm{H}$, citant la même Agence mais pour un tout autre tableau, écrit que « le potentiel techniquement récupérable de $180 \mathrm{TCF}$, soit 5000 milliards de $\mathrm{m}^{3}$ » fait de la France « le pays le mieux doté en Europe avec la Pologne » (p.9). Certes, peu après, il émet une forte réserve :

21. Il convient toutefois d'insister sur le caractère de simple évaluation théorique de ces chiffres, qui se fondent sur les connaissances actuelles du sous-sol et sur des forages peu nombreux. (H, p.10)

Dans ces trois pages sur les ressources en hydrocarbures présentées comme « un enjeu potentiellement considérable », les connecteurs concessifs et les modalisateurs d'incertitude - " il pourrait s'agir ", " la France possèderait ", " semble liée ", " devraient être inférieures »-sont nombreux et peuvent donner l'impression d'une grande objectivité de la part du rapporteur. Mais les réserves émises sont suivies de trois paragraphes insistant sur la " facture énergétique de la France » et amenant le lecteur à penser qu'aucune ressource n'est à négliger. De plus, le dernier énoncé combat la prudence d'une source insistant sur les coûts d'exploitation probablement élevés du gaz de schiste :

22. Votre rapporteur observe toutefois que la production d'une quantité importante de gaz de schiste naturel en France permettrait de réduire fortement les coûts liés au transport. $(H, p .11)$

On voit ainsi que le sénateur fait tout dans ce passage pour soutenir l'idée d'importantes ressources tout en préservant les apparences de l'impartialité. HC, pour leur part, abordent la question des réserves en deux temps : après un bref paragraphe dans la première partie introduit par le titre à orientation négative cité sous (18), ils y reviennent, au début de leur deuxième partie consacrée au code minier, en utilisant le mot «tentation » (voir exemple 9) qui indique une nette prise de distance vis-à-vis d'une possible exploitation de ces gisements. De fait, passant en revue les ressources potentielles du Bassin parisien et celles du sud-est, HC concluent :

23. Le rapport du CGIET et du CGEDD envisage des réserves exploitables dans le sud-est du pays de l'ordre de 500 milliards de mètres cube, en se fondant à nouveau sur des comparaisons géologiques. // Une nouvelle fois, vos rapporteurs soulignent que ces chiffres, très impressionnants de prime abord, ne sont que des estimations. (HC, p.24)

Outre que les 5000 milliards d'Houel ont été réduits d'un facteur 10 par une restriction au seul sud-est, le syntagme « une nouvelle fois » sous-entend que les promoteurs du gaz de schiste ne sont pas avares de tels chiffres et le dernier énoncé met en lumière la sagacité critique des rapporteurs qui ne se laissent pas impressionner par de simples « estimations ». 
GM, quant à eux, commencent par citer le paragraphe entier d'HC dont le premier énoncé de (23) constitue la fin, puis ajoutent :

24. Comme leurs collègues avant eux, vos rapporteurs considèrent ces chiffres pour une simple suspicion de l'existence de la ressource, et non pour une preuve de sa présence dans le soussol français. C'est aussi, du reste, la position de l'agence internationale de l'énergie qui accueille avec beaucoup de prudence ces estimations. (GM, p.36)

On voit que l'expertise de l'AIE est ici mise au service de la prudence, là où $H$ l'utilisait dans un but opposé. Dans le chapeau introductif de cette sous-partie, GM se sont montrés encore plus critiques :

25. Les chiffres qui circulent - et qui sont le fait des administrations américaines - à propos des ressources potentielles du sous-sol français interrogent. Alors qu'aucune exploration n'a été menée à son terme sur le territoire national et que les connaissances géologiques sont bien inférieures à celles des services des Etats-Unis sur leur sous-sol, la méthode de calcul interpelle. Il semble qu'elle procède simplement d'une comparaison entre les caractéristiques des sols européens et les propriétés des formations géologiques les plus riches d'outreAtlantique. (p.34)

Cette prise de position liminaire qui souligne l'absence d'exploration et présente les estimations comme de simples extrapolations affaiblit considérablement la portée des chiffres énoncés ensuite. Cet éclairage favorise la conclusion ${ }^{15}$ de Martin - qui souhaite renoncer à l'exploitation - mais oblige en revanche Gonnot, qui, lui, y est favorable, à revenir dans sa conclusion sur la question des réserves avec une nouvelle formulation:

26. Je suis donc persuadé que cette interdiction ne sera que temporaire. Comment pourrait-il en être autrement si notre sous-sol renferme vraiment les richesses en hydrocarbures que disent les experts ? Elles sont, affirment-ils, les deuxièmes par leur importance en Europe, derrière celles de la Pologne. (p.101)

Même si la prudence à l'égard des experts demeure, le dernier énoncé fait écho à celui d'Houel vu plus haut. Dans le débat sur les réserves potentielles, nous voyons ainsi que les trois rapports orientent différemment l'interprétation par la contextualisation divergente de sources pourtant identiques (Agence internationale de l'énergie et CGIET-CGEDD). Les discours autres sont tantôt relayés avec enthousiasme, tantôt mis à distance, le lexique se charge de subjectivité et l'ordre des énoncés explique des inversions argumentatives d'un rapport à l'autre, tel argument concédé par Houel devenant un argument phare chez HC ou GM.

Toujours en ce qui concerne le rapport aux chiffres, dont Bacot, Desmarchelier et RémiGiraud (2012) ont rappelé l'usage complexe en politique, on observe que HC et GM mettent en garde leurs lecteurs concernant la quantité apparemment réduite d'additifs chimiques dans l'eau utilisée pour la fracturation : en effet, comme l'illustre dans le rapport $\mathrm{HC}$ un diagramme produit par IFP-énergies nouvelles, les additifs ne constituent que $0,14 \%$ du fluide de fracturation qui est composé d'eau à $94,82 \%$, de sable à 5,24\%. Mais, précisent $\mathrm{HC}$ :

27. Vos rapporteurs tiennent à mettre en garde contre une interprétation rapide des pourcentages affichés : le fluide de fracturation nécessaire à une opération varie de 5000 à 20000 mètres cube selon la ressource recherchée. Cela correspond ainsi à un volume de plusieurs dizaines de mètres cube pour un puits. (HC, p.19)

GM vont encore plus loin lorsqu'ils écrivent :

28. Historiquement, la communication des opérateurs pétroliers aux Etats-Unis s'est articulée autour de plusieurs axes afin d'éviter une hostilité populaire croissante. En premier lieu, la composition des fluides de fracturation est toujours présentée au profane sous la forme de pourcentages. Ceux-ci font apparaitre une forte prédominance de l'eau (de l'ordre de 95\%), un complément de sable (approximativement 4\%) et une quantité négligeable de produits chimiques, inférieure à $1 \%$. Toutefois, il faut garder à l'esprit que le besoin en eau pour un 
puits dépasse les $10000 \mathrm{~m}^{3}$ : la masse d'additifs employés s'exprime par conséquent en dizaine de tonnes. (GM, p.48)

Dans ce passage, la présentation sous forme de pourcentage est clairement envisagée comme une stratégie de dissimulation de la part des opérateurs pétroliers. Les graphiques, chiffres et tableaux, très présents dans les rapports, ne signifient donc pas nécessairement un rapport de confiance aveugle aux données fournies par l'industrie pétrolière. Mais il est à noter qu'Houel n'évoque pas du tout le volume des adjuvants chimiques. Il mentionne dans les risques environnementaux la consommation d'eau et la présence d'additifs mais sans plus de précision et en renvoyant dos à dos les pro- et contre gaz de schiste :

29. De nombreux dommages à l'environnement et nuisances diverses ont été allégués à l'égard de l'exploitation des hydrocarbures non conventionnels [suit l'énumération]. La plupart de ces allégations font l'objet de controverse entre les industriels et l'administration américaine et ceux qui dénoncent l'exploitation des gaz de schiste ou les conditions de cette exploitation. (H, p.12)

L'emploi des lexèmes allégués et allégations - qui lui sont propres - minimise considérablement la portée des critiques en les présentant comme sujettes à discussion. La partie sur les risques environnementaux est très réduite dans ce rapport : elle représente $9,5 \%$ des occurrences, contre $22,3 \%$ pour $\mathrm{HC}$ et $36,3 \%$ pour $\mathrm{GM}$, indiquant un parti-pris pro-gaz de schiste. Cette minoration ressortit à une stratégie argumentative claire (voir Perelman et Obrechts-Tyteca $2008: 155$ ).

\subsection{Quelle protection de l'environnement ?}

Bien qu'HC aient sans nul doute contribué fortement, par les amendements qu'ils ont conseillé d'apporter à la loi Jacob, à un cadrage restrictif du débat sur le gaz de schiste à la seule question de la fracturation hydraulique, il n'en reste pas moins que les pages qu'ils consacrent aux risques environnementaux entérinent sans restriction tous les griefs que les anti-gaz de schiste ont soulevés lors des manifestations populaires et qui entrent en conflit avec la demande de classement par l'UNESCO des sites des Causses et Cévennes ${ }^{16}$.

Le premier paragraphe du rapport nous éclaire d'emblée sur la position de ses auteurs ${ }^{17}$ :

30. La réalisation de nos objectifs [en matière d'énergies respectueuses de l'environnement], il faut le reconnaitre, sera bien entendu difficile. Est-ce pour autant qu'il faut se ruer sur les gisements d'énergies fossiles dès leur découverte, sans se préoccuper des risques environnementaux et sanitaires que leur exploitation génère? $(\mathrm{p} .7)$

L'avis favorable à la proposition de loi Jacob va se fonder sur la prise en compte des risques environnementaux. Dans les pages de la sous-partie consacrée à ces risques, les lexèmes suscitant l'inquiétude - « si l'opération est mal maitrisée », " des plus dangereuses », " pollutions irrémédiables »-, ne sont pas rares, même s'ils sont la plupart du temps modalisés par des expressions telles que « peuvent constituer », « peut entrainer ». Ces lexèmes à orientation négative sont notamment présents dans plusieurs sous-titres tels que «Les nuisances marquées lors de la phase initiale de production : noria de camions et emprise au sol. » (HC, p.20). À propos du transport de l'eau, HC écrivent après une longue énumération : « la liste des dommages est longue » (p.20).

Cette tendance est encore renforcée chez GM où sont passés successivement en revue quatre types de risques : emprise sur l'espace, usage de produits chimiques, risques de contamination de l'eau, rejets dans l'atmosphère. Chaque sous-partie, plus détaillée que dans le rapport $\mathrm{HC}$, explique la nature des risques, passe au crible les affirmations des industriels, fait état des mesures prises aux États-Unis ou au Canada pour éviter les effets indésirables ou empêcher que des incidents ne se renouvellent, présente le cas échéant les conclusions d'études scientifiques et se conclut sur des recommandations au législateur dans le but de minimiser au maximum les risques en cas d'exploitation. Le choix de soustitres interrogatifs - « des puits à n'en plus finir ? », « des produits dangereux ? », « une eau recyclée ? »- construit un éthos d'impartialité et permet parfois d'écarter des risques 
imaginaires (notamment les risques sismiques), mais chaque sous-partie se conclut dans la plupart des cas par l'affirmation de risques avérés et insiste sur un devoir de vigilance.

Une autre caractéristique de la position de GM est leur méfiance explicite à l'égard des industriels pour ce qui est du souci de l'environnement. Ils ne cessent d'insister, dans l'hypothèse où l'on autoriserait l'exploitation en France, sur la nécessité de la réglementer de façon «draconienne » (p. 41), sans s'en remettre à l'autorégulation par les entreprises :

31. Les opérateurs demeurent des entreprises privées dont la vocation consiste à maximiser le profit et donc, aussi, à minimiser les investissements de précaution. L'approche d'autorégulation qui a prévalu aux États-Unis ne peut être acceptée sur le territoire national. (GM, p.41)

Alors qu'il a une taille comparable au rapport du CGIET-CGEDD, le rapport GM utilise les lexèmes contamination, contaminer, polluant, pollution et polluer 39 fois là où le rapport des experts ne les emploie que 22 fois. Même un lexème plus neutre tel que protection (protection de l'espace, de l'eau) est susceptible de faire élaborer aux lecteurs un schéma actantiel ${ }^{18}$ où, face à des industriels préoccupés par leur profit et constitués en opposants, l'État doit poursuivre une quête qui est celle de la « préservation de l'environnement » (titre de la deuxième partie) en ayant pour adjuvant une réglementation qui ne néglige aucun détail.

Outre cette insistance sur la réglementation, GM se distingue aussi d'HC et $\mathrm{H}$ par les garants invoqués à l'appui de leurs recommandations. Cette question est importante car les rapporteurs fondent leurs préconisations sur des autorités : l'action publique est présentée comme guidée par des impératifs énoncés dans des textes juridiques.

HC placent explicitement leur rapport sous l'égide de la Charte de l'environnement, dont l'article 6 est mis en exergue de leur introduction :

32. «Les politiques publiques doivent promouvoir un développement durable. À cet effet, elles concilient la protection et la mise en valeur de l'environnement, le développement économique et le progrès social. »

La Charte crée une obligation de « protection de l'environnement » qu'HC estiment incompatible avec l'exploitation du gaz de schiste par fracturation hydraulique. La Charte apparait comme un cadre normatif qui guide la prise de décision et permet de hiérarchiser des impératifs également légitimes :

33. La sécurisation des approvisionnements énergétiques est un enjeu majeur, auquel il ne faut pas sacrifier nos valeurs. (HC, p.10)

Or la Charte est à nouveau évoquée dans la dernière partie du rapport pour justifier les explorations :

34. À ce titre, devrait être sollicitée l'expertise d'organismes publics comme le BRGM, IFPÉnergies nouvelles ou INERIS en vue de l'identification " des meilleures techniques disponibles ", conformément à l'article L. 110-1 du code de l'environnement ou de la recherche de nouvelles technologies conciliant efficacité industrielle et conformité à la Charte de l'environnement. (p.30)

Cette préconisation conduira la commission à ajouter à la loi un article prévoyant « la remise par le Gouvernement d'un rapport annuel au Parlement sur l'évolution des techniques d'exploration et d'exploitation et la connaissance du sous-sol » (p. 72), article s'appuyant à nouveau sur l'article 6 de la Charte, cité à la p. 73. Houel est plus explicite encore lorsque, à l'appui de l'amendement proposé par HC, il affirme : 
35. Le développement durable doit donc être considéré dans ses trois piliers, le développement économique et le progrès social étant eux aussi tout particulièrement concernés par l'approvisionnement en énergie. (H, p.19, c'est l'auteur qui souligne)

On reconnait là toute l'ambigüité, bien soulignée par Krieg-Planque (2010), de la formule « développement durable » qui prétend concilier des inconciliables. La discussion de la commission de l'Assemblée Nationale montre qu'il s'agit là d'un point sensible, et contesté par ceux qui, à l'instar du député écologiste Yves Cochet, souhaitent interdire toute nouvelle exploitation d'énergies fossiles :

36. Qu'il s'agisse d'hydrocarbures conventionnels ou non, la pollution de l'atmosphère par le carbone est la même, même si la magie du greenwashing nous convainc de la possibilité de recourir à une technique d'extraction propre. (HC, p.37)

La question des émissions de gaz à effet de serre, qu'HC envisagent mais sans la prendre comme critère essentiel de décision, va devenir un point central dans le troisième rapport et expliquer la divergence entre Gonnot et Martin. Gonnot plaide pour une interdiction « temporaire » qui obéisse au principe de précaution mais qui préserve la possibilité de remplacer plus tard les importations d'hydrocarbures par une production nationale. Il conclut ainsi son avis personnel :

37. On peut admettre que la France veuille prendre le temps nécessaire à se garantir de toute menace sur l'environnement et la santé publique. [...] J'espère, en attendant, que le dossier des gaz et huile de schiste restera ouvert pour être traité demain sans passion, mais avec sérieux et méthode comme ont essayé de le faire vos deux rapporteurs pendant cent jours, audelà de leurs divergences politiques... (GM, p.102-103)

Pour Martin, outre la prévention des atteintes à l'environnement et le respect de l'avis des citoyens, l'interdiction de l'exploitation s'impose au nom de la transition énergétique :

38. La France doit enfin renoncer aux gaz de schiste - c'est la raison la plus importante à mes yeux - car, dans le cas contraire, elle tournera le dos à la nécessaire transition environnementale qu'impose l'état de la planète et celui de nos ressources naturelles. (GM, p.106)

Il s'agit pour lui de " faire le choix des générations futures en disant non à une ressource incertaine, anti-écologique »(p.107). La valeur de préservation de l'avenir se trouve incarnée pour lui dans des textes juridiques contraignants auxquels il se réfère à plusieurs reprises :

39. D'une manière plus générale, la France doit s'interdire une activité qui ne lui permettrait pas de respecter ses engagements nationaux (loi POPE), européens (« Paquet énergie climat ») et internationaux (protocole de Kyoto et engagements de Copenhague et de Cancun à stabiliser de $2{ }^{\circ} \mathrm{C}$ le réchauffement climatique). (GM, p.106)

Face aux quatre autres rapporteurs qui incluent la croissance économique dans leur définition du développement durable et réduisent la protection de l'environnement à l'élimination de nuisances, Martin juge l'exploitation du gaz de schiste illégitime car elle entre en contradiction avec les objectifs de limitation du réchauffement climatique.

\section{Conclusion}

Cette étude comparée à la fois quantitative et qualitative de trois rapports parlementaires montre que députés et sénateurs s'emploient à construire dans leurs rapports un éthos de conviction et d'impartialité : ils assument pleinement leur mission prescriptive et évaluative tout en prenant soin de montrer une distance critique vis-à-vis des sources qu'ils ont pu 
consulter. Cette distance critique est néanmoins dépendante de leurs convictions propres et s'exerce préférentiellement sur les éléments factuels qui pourraient aller à l'encontre de leur orientation argumentative, comme le montre la façon différente dont les trois rapports reprennent les données disponibles sur les réserves en gaz de schiste. Si ce rapport aux chiffres montre une différence nette entre $\mathrm{H}$ d'une part, HC et GM d'autre part, la comparaison de $\mathrm{HC}$ et GM sur le plan argumentatif dégage d'autres différences : alors qu'ils ont une vision similaire des risques environnementaux, ils ne les évoquent pas avec les mêmes objectifs. Pour HC, ces risques sont la raison essentielle pour surseoir à l'exploitation alors que, pour GM, ils justifient un vigoureux plaidoyer en faveur d'une réglementation stricte des activités industrielles. Enfin, la protection de l'environnement invoquée dans les trois rapports n'est pas comprise de la même façon par les cinq parlementaires : pour quatre d'entre eux, elle recouvre essentiellement le contrôle des risques de pollution dus aux activités extractives, mais pour Philippe Martin, elle implique la prise en compte des impératifs de la lutte contre le réchauffement climatique et donc l'abandon définitif de toute extraction.

Ce corpus éclaire d'un jour nouveau les réflexions en analyse du discours sur l'expression des désaccords dans les textes institutionnels et sur les relations entre discours politique et discours d'experts. L'éthos parlementaire engagé fait du rapport d'information parlementaire un type de rapport où le dissensus apparait de façon peut-être plus explicite que dans des rapports émanant d'experts. Il faudrait cependant vérifier si cette caractéristique s'observe aussi sur des sujets d'une actualité moins brûlante que l'exploitation du gaz de schiste dans le contexte de 2011.

Du point de vue méthodologique, il nous semble nécessaire, pour rendre compte de la mobilisation des ressources verbales dans ces rapports, d'articuler la dimension énonciative et la dimension argumentative des discours et de mobiliser la notion d'éclairage élaborée par Grize dans sa logique des langues naturelles. L'étude argumentative et l'attention à la matérialité verbale permet de nuancer sur certains aspects l'approche sociologique de Chailleux qui tendait à minorer les différences entre les rapports et à négliger certaines de leurs orientations. Nous espérons ainsi avoir montré l'intérêt d'une collaboration interdisciplinaire entre sociologie de l'action publique et analyse de discours pour étudier des textes souvent un peu négligés par cette dernière discipline.

\section{Corpus}

Rapport Havard-Chanteguet $\left(\mathrm{N}^{\circ} 3392\right)$. Mai 2011. URL: http://www.assembleenationale.fr/13/rapports/r3392.asp

Rapport Houel (N556). Juin 2011. URL : http://www.senat.fr/rap/110-556/110-5561.pdf

Rapport Gonnot-Martin (N³517). Juin 2011. URL : http://www.assemblee-nationale.fr/13/rapinfo/i3517.asp

Rapport OPECST (Nº174). Novembre 2013. URL : http://www.senat.fr/rap/r13-174/r13174 mono.html

Rapport Barbier (N¹919). Avril 2014. URL : http://www.assemblee-nationale.fr/14/rapinfo/i1919.asp

Rapport Bataille-Schneider (N²747). Mai 2015. URL : http://www.assemblee-nationale.fr/14/rapinfo/i2747.asp

\section{Références bibliographiques}

Adam, J.-M. (2011). Les textes. Types et prototypes. Paris : Armand Colin. Amossy, R. (2010). La présentation de soi. Éthos et identité verbale. Paris : PUF.

Authier-Revuz, J. (2020). La représentation du discours autre. Principes pour une description. Berlin : De Gruyter. Livre en open access. 
Bacot, P., Desmarchelier D. et Rémi-Giraud S. (2012). Le langage des chiffres en politique, Mots. Les langages du politique, 100 , p. 5-14.

Chailleux, S. (2016). Incertitude et action publique. Définition des risques, production des savoirs et cadrage des controverses. Revue internationale de politique comparée, 2016/4, n²3, 519- 548.

Chailleux, S. (2019). Le rapport, un instrument d'action publique. La controverse sur le gaz de schiste au prisme des rapports officiels. Politique et sociétés, Volume 38/2, 3-26.

Grize, J.-B. (2004). Le point de vue de la logique naturelle : démontrer, prouver, argumenter. L'argumentation aujourd'hui : positions théoriques en confrontation, M. Doury et S. Moirand, Paris : Presses de la Sorbonne Nouvelle, p.35-44.

Krieg-Plangue, A. (2010). La formule "développement durable" : un opérateur de neutralisation de la conflictualité. Langage et société $\mathrm{n}^{\circ} 134,5-29$.

Ledouble, H. et Monte, M. (2019). Analyse comparative de rapports parlementaires et scientifiques sur le gaz de schiste : contenu thématique et caractéristiques énonciatives. Babel civilisations et sociétés, $\mathrm{n}^{\circ} 17,131-166$.

Lemieux, C. (2007). À quoi sert l'analyse des controverses ? Mil neuf cent. Revue d'histoire intellectuelle, 2007/1, $\mathrm{n}^{\circ} 25,191-212$.

Maingueneau, D. (2002). Problèmes d'éthos. Pratiques, 113-114 : 55-68.

Mellet, S. et Monte M. (2008). « Néanmoins et toutefois ». Concession et dialogisme. Les connecteurs concessifs à l'épreuve des corpus, Mellet S. (dir.), 2008, Berne : Peter Lang, 55-85.

Monte, M. (2011). Modalités et modalisation : peut-on sortir des embarras typologiques ? Modèles linguistiques, tome XXXII, vol. 64, 85-101.

Née, E., Sitri, F., Veniard, M. (2014). Pour une approche des routines discursives dans les écrits professionnels. Actes $d u 4^{e} C M L F$, EDP Sciences, $2113-2124$. http://dx.doi.org/10.1051/shsconf/20140801195 (consulté le 16 décembre 2019).

Née, E., Sitri, F., Veniard, M. (2016). Les routines, une catégorie discursive pour caractériser les genres? Lidil, $\mathrm{n}^{\mathrm{0}} 53$ («Phraséologie et genres de discours »), 71-93.

Née, E., Oger C. et Sitri F. (2017). «Le rapport : opérativité d'un genre hétérogène », Mots. Les langages du politique [En ligne], $\mathrm{n}^{\circ} 114$

Oger C. et Ollivier-Yaniv C. (2006). Conjurer le désordre discursif. Les procédés de « lissage » dans la fabrication du discours institutionnel. Mots. Les langages du politique [En ligne], $\mathrm{n}^{\circ} 81$

Rennes, J. (2016). Les controverses politiques et leurs frontières. Etudes de communication, ${ }^{\circ} 47$, 21-48.

Oger, C. (2013). Discours d'autorité, discours autorisés. Faire référence et dire l'institution. Dossier d'HDR, Université Paris-Sorbonne, CELSA.

Perelman, C. et Obrechts-Tyteca L. (2008 [1958]). Traité de l'argumentation. Ed. de l'Université de l'Université de Bruxelles.

Vignes, L. (2019). « Ce ne sera pas du lobbying, juste de l'information ». Analyse discursive et actantielle du discours de la presse autour de la création du CHNC. Babel civilisations et sociétés, n¹7, 19-42.

\footnotetext{
${ }^{1} \mathrm{https} / / / \mathrm{www}$. legifrance.gouv.fr/eli/loi/2011/7/13/2011-835/jo/texte.

${ }^{2}$ On trouvera dans Chailleux 2016 une comparaison détaillée entre la controverse au Québec et en

France et un éclairage précis sur la façon dont a été cadré le débat public.

${ }^{3}$ Nous désignerons les rapports parlementaires par l'initiale de leurs auteurs, indiquée entre

parenthèses lors de la première mention : HC pour Havard-Chanteguet, $\mathrm{H}$ pour Houel, GM pour Gonnot-Martin, B pour Barbier, BS pour Bataille-Schneider, sauf celui de 2013 qui sera désigné par OPECST, le rapport des ingénieurs par le sigle CGIET-CGDD, le rapport du conseil scientifique régional d'Île-de-France par CSIDF et le rapport du Conseil des Académies canadiennes par CAC. Les références permettant d'accéder à ces rapports sont citées en annexe.

${ }^{4} \mathrm{Ce}$ nombre n'inclut pas les légendes des tableaux et graphiques.

${ }^{5}$ Le graphique met en évidence une spécificité statistique calculée par le logiciel TXM en comparant le nombre d'occurrences d'une forme dans une partie du corpus (ici dans chaque rapport) à ce qui serait attendu si sa répartition dans toutes les parties du corpus était homogène.

${ }^{6}$ Sur les graphiques les rapports sont rangés dans l'ordre alphabétique de leur source.

${ }^{7}$ Le nombre entre parenthèses indique le nombre d'occurrences.

${ }^{8}$ Dans le calcul des spécificités, on appelle zone de banalité la zone comprise entre un indice de +2 et de -2 par rapport à la fréquence théorique attendue. Il n'y a dans ce cas ni sous- ni suremploi.
} 


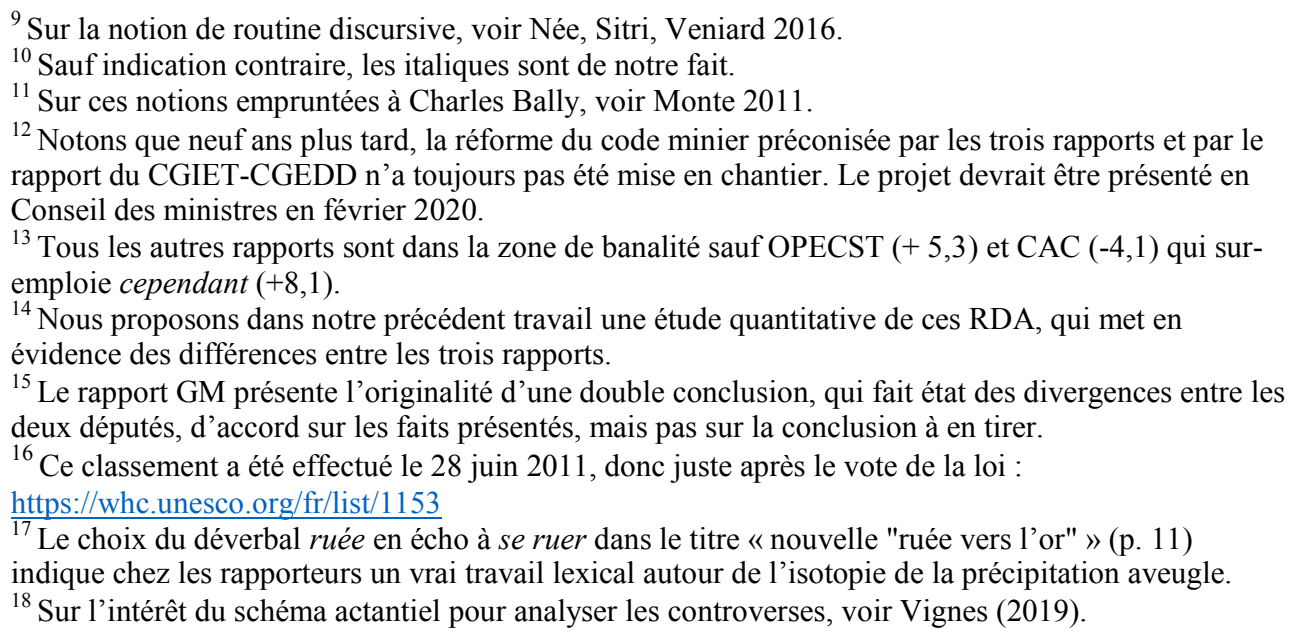

